\title{
Hepatitis E: A Literature Review
}

\author{
Juliana Ayres de Alencar Arrais Guerra*, Katia Cristina Kampa, \\ Daphne Gonçalves Benatti Morsoletto, Alcindo Pissaia Junior \\ and Cláudia Alexandra Pontes Ivantes
}

Nossa Senhora das Graças Hospital, Alcides Munhoz Street, 433 - Mercês, Curitiba - PR, Brazil

\begin{abstract}
Hepatitis $E$ is the fifth known form of human viral hepatitis. Although not very common in our clinical practice, the incidence in Western countries is increasing. Infection with the hepatitis $\mathrm{E}$ virus (HEV) may be related to acute illness, liver failure, chronic hepatitis and cirrhosis. HEV itself is an RNA virus, with eight described genotypes (HEV 1-8), four of which more commonly affect humans and have, thus, been better studied. Besides liver manifestations, genotype 3 is also related to extra-hepatic manifestations, such as neurological, renal and rheumatological. Evolution to chronic disease occurs especially in patients who underwent transplantation, have hematological malignancies requiring chemotherapy, or have infection with the human immunodeficiency virus. The diagnosis may be difficult because of the low availability of tests and due to low sensibility and specificity. The acute form of illness does not have to be treated, but the chronic one does. We present here a literature review of hepatitis $E$ and the relation between chronic hepatitis $\mathrm{E}$ and transplantation.

Citation of this article: Guerra JAAA, Kampa KC, Morsoletto DGB, Pissaia Junior A, Ivantes CAP. Hepatitis E: a literature review. J Clin Transl Hepatol 2017;5(4):376-383. doi: 10. 14218/JCTH.2017.00012.
\end{abstract}

\section{Introduction}

Hepatitis $E$ is the fifth known human viral hepatitis and is probably the most common cause of acute viral hepatitis in the world. ${ }^{1-3}$ Despite being an important cause of hepatitis and being widely studied, the hepatitis $E$ virus (HEV) remains poorly understood, with little comprehension about its mechanisms of replication and pathogenesis. ${ }^{4}$ The origin of hepatitis $\mathrm{E}$ also remains unknown. While some have theorized that it is an emerging disease, historical records suggest that hepatitis $\mathrm{E}$ may be old. ${ }^{5}$ It was first identified as a non-A, non-B hepatitis virus in 1980. In 1983, the Russian virologist Mikhail Balayan visualized the virus through electron

Keywords: Hepatitis E; Chronic hepatitis E; Immunosuppression; Transplantation.

Abbreviations: ALT, alanine aminotransferase; CSF, cerebrospinal fluid; FDA, Food and Drug Administration; HEV, hepatitis E virus; ORF, open reading frame SOF, sofosbuvir.

Received: 24 February 2017; Revised: 27 June 2017; Accepted: 20 July 2017

*Correspondence to: Juliana Ayres de Alencar Arrais Guerra, Nossa Senhora das Graças Hospital, Alcides Munhoz Street, 433 - Mercês, Curitiba - PR 80810-040, Brazil. Tel: +55-41-3240-6060, E-mail: juliana.arrais.med@gmail.com microscopy when examining his own feces after selfadministration of contaminated material. ${ }^{1,5}$

The incidence of acute hepatitis $E$ is estimated at 3 million human cases per year worldwide, with around 70,000 deaths. ${ }^{6}$ Most cases occur in endemic countries, but the number of cases in low-endemic areas has increased. ${ }^{6} \mathrm{HEV}$ seroprevalence is high in developing countries, such as India and Southeast Asia, ranging from $27-80 \% .{ }^{1,6}$ Surprisingly, some studies in developed countries, such as the United States of America and the United Kingdom, have shown an unexpectedly high seroprevalence (of 21-25\%) and have indicated the possible reasons for such as subclinical infection, animal exposure, cross-reactivity with other agents or false positive test results. ${ }^{6}$ Acute disease mortality is $1-4 \%$, with risk being higher in pregnant women and immunodeficient patients. ${ }^{6}$

\section{Virology}

HEV is a small non-enveloped virus, $27-34 \mathrm{~nm}$ in diameter, with a single-stranded RNA genome. ${ }^{1,7,8}$ It replicates in the cytoplasm of cells, ${ }^{1,8,9}$ and can replicate in hepatocytes, small intestine and colon cells, and lymph nodes. ${ }^{9}$ It presents three discontinuous open reading regions-called Open Reading Frames (ORF)-and ORFs 2 and 3 can overlap each other. $^{8,10}$ Of these regions, ORF1 is the largest, containing several conserved domains, and encodes non-structural proteins. ORF2 encodes the viral capsid protein, whereas ORF3 encodes a small phosphoprotein, with uncertain function. ${ }^{7}$ The capsid protein is highly immunogenic and antibodies against it neutralize and are protective. Thus, the capsid antigen is the preferred protein for the development of vaccines. $^{5}$

Currently, HEV is included as a member of the family Hepeviridae, which includes two genera: Orthohepevirus and Piscihepevirus. The genus Orthohepevirus encompasses all mammalian and avian HEV variants and is subdivided into four species: A-D. Moreover, among the Orthohepevirus $A$ species, eight genotypes are recognized: HEV $1-8^{1,5-7,9,11}$ (Table 1). Recently, Woo et al. ${ }^{12}$ performed a study in the United Arab Emirates, in which they analyzed 203 fecal samples of adult dromedaries (Camelus dromedarius). The authors described HEV RNA found in these fecal samples and showed the ability of these to infect humans, indicating a previously unknown potential reservoir and source of HEV infection for humans. ${ }^{12}$ This finding was also supported by Lee et al., ${ }^{13}$ who reported a case of chronic infection with camelid HEV in a liver transplant recipient who regularly consumed camel meat and milk. 
Guerra J.A.A.A. et al: Hepatitis E - literature review

Table 1. Currently classification of HEV

\begin{tabular}{llll}
\hline Family & Genera & Species & Genotypes \\
\hline Hepeviridae & Orthohepevirus & A, B, C, D & I, II, III, IV, \\
& & & V, VI, VII, \\
& & & \\
& & & \\
& Piscihepevirus & & \\
\hline
\end{tabular}

HEV grows poorly in vitro and this hinders the comprehension of its cellular cycle. However, recently, several cell culture systems have been developed for HEV genotypes 3 and $4 .{ }^{8} \mathrm{An}$ important finding was the identification of short human sequence inserts into the virus' RNA, which facilitated the adaptation of tissue culture. ${ }^{1}$ Notably, similar insertions were identified in isolated RNA directly from patients with severe neurological complications of hepatitis $E{ }^{1}$ These results suggest that recombination events can alter the replication capacity, tissue specificity and pathogenicity of the virus, making it an unique agent among the viruses of hepatitis. ${ }^{1}$ Liver disease caused by HEV infection is primarily a consequence of immune, humoral and cellular responses, since HEV is, in most circumstances, non-cytopathic. ${ }^{14}$ Despite the various genotypes, the various species of HEV can be considered as belonging to the same serotype. ${ }^{15}$

Genotype 1, present in Asia and North Africa, has been the leading cause of waterborne epidemics and significant sporadic disease. ${ }^{5,7}$ Genotype 2, however, caused a single epidemic in Mexico and several epidemics in Central Africa. ${ }^{5}$ Genotype 3, present in North and South America, several countries in Europe, Japan and few Pacific countries, ${ }^{5}$ is considered a reemerging zoonosis. ${ }^{6}$ Finally, genotype 4 , responsible for sporadic cases, ${ }^{6}$ is present in parts of China, Japan, Taiwan and Vietnam, but has also been reported in Europe. ${ }^{5,6}$

The four more prevalent genotypes are allocated into two groups. ${ }^{1}$ Epidemic hepatitis E includes genotypes 1 and 2, which are considered human viruses and have caused the epidemics of hepatitis. These forms are transmitted mainly by contaminated water and the fecal-oral route. Autochthonous/endemic hepatitis $E$ includes genotypes 3 and 4, which are considered swine viruses (common in domestic and wild pigs), capable of infecting humans as an accidental host and therefore considered zoonotics. They do not seem to cause disease in pigs, however, but instead only infecting them. ${ }^{5}$ Chronic hepatitis $\mathrm{E}$ cases are usually caused by genotype 3 virus, although there is a report of a case of chronic hepatitis $E$ in children caused by genotype $4 .{ }^{6,16}$

In addition, epidemiology and clinical disease seem to be associated with the molecular structure of the virus, justifying this territorial division of occurrence of the different virus genotypes. ${ }^{2}$ There would be cross-neutralization expected between the four genotypes, despite having different clinical outcomes and epidemiology. ${ }^{1}$ Evidence for this cross-immunity was provided by a study in China in which 100,000 participants were immunized with a vaccine against genotype 1 ; although China represents a region where genotype 4 predominates, the study demonstrated that the infection was prevented. ${ }^{14}$

\section{Epidemiology}

Based on seroprevalence, it is estimated that one-third of the world's population has been infected with HEV. ${ }^{15}$ The mean incubation time of HEV is 40 days, and the highest rate of involvement is between 15 and 40 years of age. ${ }^{15}$ It affects more men than women, with a ratio of $2: 1$ in developing countries and $>3: 1$ in developed countries. ${ }^{9}$ In Brazil, data on the seroprevalence of HEV are limited. Although the country is classified as a moderate endemic region for hepatitis E, most studies are old or cannot be compared adequately because they have a small number of different cases or methodologies. ${ }^{17}$ Furthermore, there is the fact that screening for HEV is not routinely performed in the country, even in cases of unexplained elevation of transaminases, and that few laboratories have anti-hepatitis E tests available. ${ }^{17}$ Passos-Castilho et al. ${ }^{17}$ retrospectively evaluated all hepatitis E tests performed in a large laboratory in São Paulo between 1998 and 2013. They concluded that the rates of seroprevalence and detection of HEV appear to have increased in recent years and suggest that hepatitis $E$ should be considered in the differential diagnosis of hepatitis in Brazil. ${ }^{17} \mathrm{~A}$ study with foods derived from pork in Brazil found $36 \%$ positivity for HEV (genotype 3 ), which may be a possible source of contamination. ${ }^{18}$

Anti-HEV rates in the general population are lower in Europe and the United States than in Asia and Africa. However, research between 1988 and 1994 indicated that $21 \%$ of adults in the US had anti-hepatitis E antibody, ${ }_{1}^{1,6}$ while $38.3 \%$ had anti-hepatitis A virus positivity, $5.7 \%$ had antihepatitis $B$ virus positivity and $2 \%$ had anti-hepatitis $C$ virus positivity. ${ }^{1}$

In regard to transmission, HEV is excreted in the stool of infected persons and is transmitted by the following routes: ${ }^{7,19}$ fecal-oral, from potable water contamination; ingested food, from infected animals (raw or uncooked pig, wild boar, deer meats or entrails); zoonosis, from human exposure to body fluids of infected animals; transfusion of contaminated blood products; ${ }^{20}$ vertical transmission (maternalfetal); and transplantations with HEV-infected grafts. Of these, the most common is through contaminated water. ${ }^{4}$ However, it is often not possible to establish the route of transmission, especially in regions of low endemia and in sporadic cases in hyperendemic regions. There are zoonotic reservoirs among swine, wild boars, deer and camels. ${ }^{12,13,21}$

There are two distinct epidemiological patterns of hepatitis $E$ in different geographic regions. ${ }^{7}$ In the first, hyperendemic regions, outbreaks are large, affecting hundreds to thousands of people and the source is usually contaminated water. ${ }^{7}$ These regions include developing countries, wherein hepatitis E occurs as both sporadic and epidemic diseases, most which is caused by genotype 1 . The reported rates of anti-HEV antibodies in adults in these areas are 30 to $80 \% .{ }^{1}$ In these regions, the disease usually affects adolescents and young adults. ${ }^{1}$ Here, mortality is higher among pregnant women. ${ }^{1}$ On the other hand, due to rapid industrialization and improvement in sanitary conditions in many parts of East Asia, zoonotic HEV transmission is assuming an increasing importance with a resultant genotype switch from HEV1 to HEV3 or HEV4. ${ }^{22}$

In the second, low endemic regions, zoonotic transmission plays an important role. These regions include developed countries (the Americas and Europe), where hepatitis $\mathrm{E}$ occurs as isolated cases and small outbreaks, which have been attributed to exposure to pigs and consumption of undercooked pork. ${ }^{1}$ These cases are usually caused by genotypes 3 and 4 , which appear to be less virulent. ${ }^{5}$ This fact would explain the high prevalence of anti-HEV antibodies in 
developed countries with few reported cases of disease, since these strains probably cause clinical disease rarely..$^{5}$ In these regions, the disease usually affects older adults, in whom mortality is higher. ${ }^{1}$

It is known that pregnant women who acquire the HEV are more prone to severe and fulminant disease, ${ }^{1,7,21}$ but the reason is not fully understood. The mortality of hepatitis $E$ in this population reaches $20 \%,{ }^{3,5,9,15}$ usually in the third trimester. ${ }^{9}$ Death occurs due to obstetric problems, including hemorrhage or eclampsia, or due to fulminant hepatic failure. ${ }^{9}$ Transmission of HEV from mother to fetus, with fetal death, is common. ${ }^{5,9}$ The mortality excess in pregnancy with genotypes 1 and 2 is unique. It is not seen in cases from either genotypes 3 or 4 , although there have been some documented cases in pregnant women, nor in cases involving other hepatotropic viruses. ${ }^{9}$ Evaluation of micronutrient status and serum cytokine levels in pregnant women has suggested that nutritional and immunological characteristics play a role in this susceptibility. ${ }^{1}$

Several genetic mutations in the progesterone receptor have been associated with maternal and fetal mortality in pregnant women infected with HEV. ${ }^{14}$ Moreover, differences in immunological and hormonal responses in pregnant women with hepatitis $\mathrm{E}$ and fulminant hepatic insufficiency have been found, and recent studies have shown that the viral load of HEV in pregnant women is much higher than that in non-pregnant women. ${ }^{9}$ Different from cases of hepatitis $A$, in hepatitis $E$ cases it is unclear whether there is an association between the age at which infection occurs and the severity of the clinical presentation. ${ }^{5}$ Some authors have asserted that the severity of the disease increases with age. ${ }^{15}$ Besides, population surveys have shown that antiHEV positivity rates increase with age, namely $<10 \%$ between $6-19$ years old and $>40 \%$ in older than 60 years old. ${ }^{1}$

It was demonstrated, surprisingly, that the risk factors for the presence of hepatitis $E$ antibodies were different from the other types of viral hepatitis. For example, anti-hepatitis $\mathrm{E}$ antibody levels were lower in blacks $(14.5 \%)$ than in nonHispanics $(22.1 \%)$. There were also lower rates in men who had sex with men $(23.1 \%)$ than in those who did not $(23.9 \%)$, men who had sex with human immunodeficiency virus (HIV)-positive men (12.8\%) versus men who had sex with men without HIV (19.2\%), cocaine users $(16.8 \%)$ compared to non-users (23.6\%), women (20.4\%) compared to men $(21.6 \%)$, and others. ${ }^{1}$

The mortality of acute hepatitis $\mathrm{E}$ is $1-4 \%{ }^{1,3,5-7,15}$ Nonetheless, there may have bias in this data, since several studies only included hospitalized patients. In studies of population surveys in outbreaks, the observed mortality rate was $0.07-0.6 \%$. $^{7}$

\section{Clinical presentation}

The course and clinical presentation of hepatitis $E$ is highly variable. $^{7,14,23}$ The detailed mechanisms that lead to the different clinical outcomes in hepatitis $E$ are only partially understood. It is known that both viral factors (genotype and dose of inoculum) and host factors (presence of previous liver disease, pregnancy and distinct genetic polymorphisms) determine the course of infection. ${ }^{14}$ In most cases, hepatitis E causes self-limited illness, lasting from a few days to weeks, with an average of 4-6 weeks. ${ }^{23}$ However, in developed countries it can cause chronic disease with rapid progression to cirrhosis, especially in patients who are transplanted, have hematological malignancies requiring chemotherapy, or have infection with HIV. ${ }^{9}$

After an incubation period of 2 to 6 weeks, the common symptoms of hepatitis appear, such as fever, nausea, abdominal pain, vomiting, anorexia, malaise and hepatomegaly. ${ }^{9,23}$ Jaundice occurs in about $40 \%$ of symptomatic cases in developing countries and in up to $75 \%$ of symptomatic cases in developed countries. ${ }^{9}$ The period of symptoms and jaundice may last from days to weeks. ${ }^{1}$ Additionally, hepatitis E can lead to acute liver failure and should be remembered in differential diagnosis. One cohort showed that 8 of 80 cases of acute liver failure in Europe appeared to be associated with hepatitis $E_{1}^{21,24}$ with half of these cases being initially diagnosed erroneously as drug-induced liver damage. Another study in the United States also stated that a small number of suspected cases of drug-induced liver damage may actually be caused by hepatitis $\mathrm{E}$. They suggest that hepatitis E screening tests should be considered when the pattern of the lesion is similar to viral hepatitis and when the clinical characteristics and latency are unusual. ${ }^{25}$

In hyperendemic regions, the majority of cases present with acute and self-limited jaundice, with spontaneous resolution (viral clearance within 1 to 3 months) (Table 2). This illness is often clinically and biochemically indistinguishable

Table 2. Epidemiologic and clinical features of the more prevalent and studied genotypes

\begin{tabular}{|c|c|c|c|c|}
\hline Genotypes & Epidemiology & $\begin{array}{l}\text { Region of } \\
\text { occurrence }\end{array}$ & $\begin{array}{l}\text { Route of } \\
\text { transmission }\end{array}$ & Clinical features \\
\hline 1 and 2 & $\begin{array}{l}\text { Epidemic } \\
\text { disease }\end{array}$ & $\begin{array}{l}\text { Hyperendemic } \\
\text { regions: } \\
\text { developing } \\
\text { countries }\end{array}$ & $\begin{array}{l}\text { Contaminated water } \\
\text { and fecal-oral }\end{array}$ & $\begin{array}{l}\text { - Acute and self-limited jaundice, with } \\
\text { spontaneous resolution } \\
\text { - Severe disease among pregnant women }\end{array}$ \\
\hline 3 and 4 & $\begin{array}{l}\text { Endemic } \\
\text { disease }\end{array}$ & $\begin{array}{l}\text { Low endemic } \\
\text { regions: America } \\
\text { and Europa }\end{array}$ & $\begin{array}{l}\text { Zoonosis } \\
\text { (transmission from } \\
\text { domestic and wild } \\
\text { pigs) }\end{array}$ & $\begin{array}{l}\text { - Older age, a more marked male predominance, } \\
\text { lack of severe disease among pregnant women, } \\
\text { a higher frequency of underlying liver disease or } \\
\text { alcohol use, a somewhat higher frequency of } \\
\text { non-specific symptoms, and a higher mortality } \\
\text { rate } \\
\text { - Genotype } 3 \text { can cause chronic disease, especially } \\
\text { among the immunosuppressed }\end{array}$ \\
\hline
\end{tabular}


from that caused by other hepatotropic viruses, such as hepatitis A virus or hepatitis B virus, except for its epidemiological features, such as occurrence in outbreaks, association with contamination of water sources, young age of patients and predilection for pregnant women. ${ }^{26}$ Some of these cases have prolonged cholestatic disease and few patients develop severe liver injury that manifests as subacute or acute (or fulminant) liver failure. In addition, asymptomatic infection is also common in these areas, with milder liver injury and with only non-specific symptoms resembling acute febrile viral illness without jaundice (anicteric hepatitis); liver involvement in these patients is recognized only if laboratory studies are done. ${ }^{26}$ In patients with previous chronic liver disease, there is a greater risk for poor prognosis. In those cases, the underlying chronic disease is often unknown/silent and the diagnosis is only made when hepatitis E overlaps. ${ }^{6,7}$

In low-endemic regions, most cases are recognized during investigation of unexplained hepatitis and are sporadic. ${ }^{7}$ In these areas, there are some different characteristics from those of regions of high endemicity; these include older age, a more marked male predominance, lack of severe disease among pregnant women, a higher frequency of underlying liver disease or alcohol use, a somewhat higher frequency of non-specific symptoms, and a higher mortality rate. ${ }^{26}$ Genotype 3 infections may be subclinical in young and healthy individuals, but are often symptomatic and lead to jaundice in older men or patients with significant comorbidities, with poorer prognosis. ${ }^{25}$ A review of four case-series of hepatitis E reported from low-endemicity areas, ${ }^{27}$ found that jaundice was the most common symptom, being reported in $68-86 \%$ of patients in the different series. Other common symptoms are asthenia, fever, joint and muscle pains, and abdominal pain. It was also reported that some patients also complained of headache, nausea and vomiting, loss of appetite, loss of weight, bowel disturbances and purpuric skin rash. ${ }^{27}$ Some patients with hepatitis $E$ in high-income countries had initially been diagnosed to have drug-induced livery injury. ${ }^{27}$

Moreover, in the last decade, chronic hepatitis E cases have been reported, showing persistently elevated transaminases, progressive liver injury and cirrhosis. These cases have mainly involved immunosuppressed patients, for example, those with solid organ transplant, HIV positivity with low CD4 count, or hematologic malignancies receiving chemotherapy. ${ }^{1,6,28}$ However, there are reported cases in immunocompetent individuals as well. ${ }^{1,6,29}$ Reports of chronic disease cases have almost exclusively involved infections with genotype 3, although one related case of chronic disease in a child has been reported as caused by genotype 4..$^{6,7,16}$ Extra-hepatic manifestations of hepatitis $E$ may occur in the acute and chronic phases (Table 3). Among them, neurological complications are the most common. ${ }^{6}$

In a study of 126 patients with acute and chronic hepatitis E infection, $5.5 \%$ had neurological manifestations. ${ }^{6,9}$ The manifestations described in this study were bilateral pyramidal signs, ataxia, proximal myopathy, encephalitis, cognitive dysfunction, peripheral demyelinating polyneuropathy and peripheral pain sensory neuropathy. In 4 patients with the chronic disease, HEV RNA was detected in the cerebrospinal fluid (CSF). Interestingly, in cloning the genetic material found in the CSF, they noticed compartmentalization of "quasispecies", suggesting that the neurological lesion in these cases must be associated with emerging neurotropic variants.
Table 3. Described extra-hepatic manifestations of hepatitis $E$

\begin{tabular}{ll}
\hline Neurological & $\begin{array}{l}\text { Bilateral pyramidal signs, ataxia, } \\
\text { proximal myopathy, encephalitis, } \\
\text { cognitive dysfunction, peripheral } \\
\text { demyelinating polyneuropathy, } \\
\text { peripheral pain sensory neuropathy, } \\
\text { Guillain-Barré syndrome, Bell's palsy, } \\
\text { acute transverse myelitis and acute } \\
\text { meningoencephalitis }\end{array}$ \\
Renal & $\begin{array}{l}\text { Membranoproliferative } \\
\text { glomerulonephritis, relapse of IgA } \\
\text { nephropathy and cryoglobulinemia }\end{array}$ \\
Rheumatologic & $\begin{array}{l}\text { Arthralgia, myalgia and skin rash } \\
\text { Pancreatic }\end{array}$ \\
Hematological & Thrombocytopenia and aplastic anemia \\
\hline
\end{tabular}

However, the mechanism and pathogenesis of neurological manifestations in hepatitis $\mathrm{E}$ are not yet known. ${ }^{30}$

Other neurological manifestations that have already been described are Guillain-Barré syndrome, Bell's palsy, acute transverse myelitis and acute meningoencephalitis. ${ }^{28,30}$ Besides, renal (membranoproliferative glomerulonephritis, relapse of IgA nephropathy and cryoglobulinemia) ${ }^{31}$ and rheumatologic complications (arthralgia, myalgia, skin rash) have also been reported. ${ }^{6}$ Finally, acute pancreatitis and hematological diseases (thrombocytopenia and aplastic anemia) have been described in association with acute hepatitis E. ${ }^{9}$

\section{Diagnosis}

Hepatitis E is an underdiagnosed disease, partly due to the use of serological tests with low sensitivity. ${ }^{9}$ Diagnosis can be made indirectly by detecting antibodies against HEV in the serum, or directly by detecting the genome of the virus in blood or other body fluids. ${ }^{9}$ There is no genotype-specific serological test. ${ }^{9}$ One study that sought to determine the kinetics of anti-hepatitis $E$ antibodies found that, at the symptom stage, anti-hepatitis $E$ antibody levels peak, then remain at these levels for 8 weeks. After, the IgM levels fall rapidly, being below the detectable level in most patients after 32 weeks. IgG levels were found to be rising already when patients were symptomatic, reaching the peak at 4 weeks after onset of symptoms and remaining at high levels for more than 1 year. The exact duration of IgG response remains unknown. ${ }^{9}$

These tests for anti-hepatitis $\mathrm{E}$ antibody screening are commercially available, but none of them has been approved by the Food and Drug Administration (FDA). Unfortunately, the sensitivity and specificity of these tests vary greatly and this could explain the discrepancies in rates of anti-hepatitis $E$ antibodies published for the various populations studied. Until tests are approved by the FDA, physicians will rely on locally available tests. The tests for viral RNA in serum and feces are confirmatory, but still experimental. ${ }^{1}$ One study compared six tests for anti-hepatitis E IgM antibodies in the serum of immunocompetent patients infected with the four types of hepatitis $E$, with sensitivity of tests between 72 and $98 \%$ and specificity between 78.2 and $95.6 \% .^{32}$ Another study evaluated two anti-hepatitis E IgM antibody tests in immunocompetent and immunocompromised patients and showed that the sensitivity was $97.7 \%$ in immunocompetent patients and 
$85-87 \%$ in immunocompromised patients, with the two tests having high specificity $(>99.5 \%) .{ }^{33}$

Trials evaluating anti-hepatitis E IgG antibodies have shown variable performance, with most available studies using serum from patients with recent infection, so that their ability to detect old/established infections remains unknown. The detection limits of these tests vary greatly and the IgG is sometimes undetectable after infection. These factors should be considered when interpreting seroprevalence data available in the literature. ${ }^{9}$ Another important point is that the concentration of anti-hepatitis E IgG antibodies could be useful in determining which level of IgG would prevent infection after natural infection or administration of the vaccine. To this end, a vaccine study suggested that the antibody concentration of $2.5 \mathrm{IU} / \mathrm{mL}$ would be protective. ${ }^{9}$

Regarding viremia, the peak occurs during the incubation period and the initial symptomatic phase. ${ }^{9}$ Hepatitis E RNA in the blood becomes undetectable about 3 weeks after the onset of symptoms but can be detected in the stool for another 2 weeks. There is no correlation between levels of viremia and intensity of symptoms. ${ }^{9}$ Thus, the initial examination for diagnosis of hepatitis $E$ should be the anti-hepatitis E IgM antibody, leaving the HEV RNA detection by RT-PCR for suspected cases with anti-hepatitis E IgM negativity, especially in the immunocompromised. $6,7,9$

Chronic hepatitis $\mathrm{E}$ is diagnosed by the detection of HEV RNA in feces or serum after a minimum of 3 to 6 months after the diagnosis of hepatitis E. Thus, IgM and IgG serological tests are not used to diagnose or exclude chronic disease. ${ }^{6}$ Very recent data, in the context of transplanted patients, found that there is no spontaneous clearance of HEV between 3 and 6 months after acute infection and this suggests that chronic infection should be considered when replication lasts more than 3 months. ${ }^{9}$ One study showed that at diagnosis, transaminases were lower in patients who progressed to chronic disease. The mean alanine aminotransferase was $300 \mathrm{IU} / \mathrm{L}$ in chronic disease and $1000 \mathrm{IU} / \mathrm{L}$ in acute disease. ${ }^{6}$ There was also no correlation found between viral serum concentration and risk of progression to fibrosis. ${ }^{6}$

Hepatic biopsies from patients with acute hepatitis $E$ show a typical pattern of portal and lobular inflammation associated with hepatocyte necrosis. Cholestasis and ductal proliferation may also be observed in varying degrees, and even cases of destructive lymphocytic cholangitis have been reported. Similar to hepatitis $C$, steatosis and plasma cells can also be found. ${ }^{6}$ In general, no distinct histological feature has been identified that allows for differentiation between hepatitis $B$ and $C$, supporting the hypothesis that the cellular immune response largely determines the severity of the disease. The inflammatory cell infiltrate in uncomplicated acute hepatitis $\mathrm{E}$ is predominantly neutrophils. ${ }^{6}$

An important differential diagnosis is drug-induced liver injury, especially in the elderly, for whom polypharmacy is common. In a more recent study from the United States, 3\% of patients with "drug-induced liver damage" were misdiagnosed as they had positive hepatitis E tests in subsequent research. Studies like this show the importance of excluding other causes of a hepatocellular lesion before making the diagnosis of drug-induced injury, especially in patients with elevated transaminases. ${ }^{9,23}$

\section{Treatment}

Several stages of the HEV cell cycle may be potential targets for development of antiviral drugs. ${ }^{8}$ Acute infection usually does not require treatment, but chronic infection should be treated by reducing immunosuppression in transplanted patients or by using antiviral therapy ${ }^{9}$ (Table 4). Chronic hepatitis $\mathrm{E}$ may lead to spontaneous resolution in some cases, but may also lead to rapid progression to cirrhosis and death. ${ }^{6}$ Hence, it is important to consider the treatment.

Kamar and colleagues ${ }^{6,9}$ demonstrated that reducing $T$ cells to target immunosuppression helped in eradicating hepatitis $\mathrm{E}$ spontaneously in transplanted patients, in up to $1 / 3$ of the cases evaluated. They reported that in the remaining $2 / 3$ of cases antiviral therapy would be indicated. All published data are based on small series and case reports, since no randomized study was performed. ${ }^{9}$ Nevertheless, one risk of reducing immunosuppression is the increased risk of rejection. ${ }^{23,28}$

A 3-month course of pegylated-interferon therapy at a dose of $135 \mathrm{~g} /$ week was conducted with 3 liver-transplanted patients and 1 hemodialysis patient who had received a kidney transplant. A sustained virological response was obtained in 3 of the 4 patients. A 12-month course of pegylated-interferon therapy was also effective in treating chronic hepatitis $E$ after liver transplantation. However, interferon cannot be used after kidney, heart and lung transplantation due to the risk of acute rejection. $^{9}$

Ribavirin, a guanosine analog, inhibits the replication of various RNA and DNA viruses. ${ }^{34}$ Studies have shown that ribavirin alone at a dose of $600-800 \mathrm{mg} /$ day for 12 weeks has led to sustained virological response in at least $2 / 3$ of chronic hepatitis $E$ cases. In addition, success with ribavirin led to its use to treat severe acute hepatitis $\mathrm{E}$, with promising results. ${ }^{1}$ Kamar et al. ${ }^{35}$ performed a study in which 59 transplanted patients (kidney, liver, heart, kidney, pancreas, lung) were treated with ribavirin at an average dose of $600 \mathrm{mg} /$ day for a median of 3 months. Fifty-four patients were genotyped and all were found to have genotype 3 infections. The researchers found that $95 \%$ of patients at the end of treatment had viral clearance, while $78 \%$ had sustained virological response. About $60 \%$ of patients had hepatitis $E$ recurrence and $40 \%$ of these patients had sustained virologic response after prolonged treatment with ribavirin. This study demonstrated that ribavirin is a good initial treatment option for chronic hepatitis $\mathrm{E}$. The main side effect of ribavirin was anemia, seen in $54 \%$ of patients, with $12 \%$ requiring blood transfusion. ${ }^{6}$

A recent systematic review evaluated the efficacy and safety of ribavirin treatment in 105 patients and of pegylatedinterferon treatment in 8 patients with chronic hepatitis $E$. Sixty-four percent of patients treated with ribavirin had an undetectable virus level within 6 months after stopping treatment, while only 2 of $8(25 \%)$ of the patients treated with pegylated-interferon achieved a sustained virologic

Table 4. Treatment of hepatitis $E$

\begin{tabular}{ll}
\hline Acute disease & - Usually does not require treatment \\
Chronic disease & - Reduction of immunosuppression in \\
& transplanted patients \\
& - Antiviral therapy: pegylated- \\
& interferon; ribavirin
\end{tabular}


response. The main side effect of ribavirin in that study was again anemia, with $35 \%$ of patients requiring erythropoietin and $10 \%$ requiring blood transfusion. On the other hand, in the pegylated-interferon group, 2 of the 8 patients developed acute transplant rejection. ${ }^{36}$

Therefore, ribavirin monotherapy has been applied, with promising results in both adults and children. The mechanism of action of ribavirin against HEV is still unknown. ${ }^{9}$ Studies with the use of sofosbuvir (SOF), a nucleotide drug against hepatitis $C$ virus, were effective in inhibiting the replication of genotype $3 \mathrm{HEV}$ in vitro, and this effect was greater when SOF was combined with ribavirin. ${ }^{37}$ However, to date, hepatitis $\mathrm{E}$ treatment is experimental, there are no guidelines, ${ }^{28}$ and neither ribavirin nor interferon have been approved for this use. ${ }^{1}$ On the other hand, Murali et al. ${ }^{6}$ suggest, as an initial approach, immunosuppression reduction and, in case of no adequate response, ribavirin at $600-800 \mathrm{mg} / \mathrm{day}$ for 3 months (with anemia monitoring) should be started.

\section{Prevention}

In the endemic areas, prevention strategies should include improving hygiene and sanitary practices. In non-endemic areas, an important measure is to avoid consumption of undercooked meat. 6,19 Two vaccines have been developed to prevent hepatitis $E$ infection. ${ }^{38}$ Shrestha et al. ${ }^{15}$ performed a phase 2 study with a recombinant vaccine with 2000 healthy adults, and found $95.5 \%$ efficacy after three doses. However, the vaccine did not progress from phase $2 .{ }^{6}$ Zhu et al. ${ }^{39}$ published results from a phase 3 , double-blind, randomized study with more than 50000 participants in each arm. Three doses of hepatitis $E$ vaccine were given at 0,1 and 6 months to participants, and the vaccine showed $100 \%$ efficacy at 12 months after vaccination. In the extension of the followup period, for up to 4.5 years, the vaccine showed efficacy of $86.8 \% .6,40$ To date, this hepatitis E vaccine garnered approval in China but has not yet been approved in other countries. ${ }^{1,38}$

Even if the vaccine becomes available, many new studies will still be needed to clarify several other questions, such as duration of protection, need for reinforcement, safety and immunogenicity in specific groups (pregnant women, patients with chronic liver disease, patients with immunogenicity), vaccine efficacy in endemic areas against genotypes 1 and 2, and vaccine efficacy in preventing and relieving symptoms after exposure to HEV. Another major obstacle will be cost. Probably, because of these and other difficulties, vaccination approval has been slow. ${ }^{38}$

\section{Chronic hepatitis E and transplantation}

Chronic hepatitis E has been described, in most cases, in immunosuppressed patients and for infection with genotype $3,1,6,9,14$ with the first case having been described in 2008. ${ }^{41,42}$ All described cases are autochthonous, and not associated with travels. ${ }^{9,43}$ Among the immunosuppressed, the cases in transplanted individuals have been most studied. ${ }^{44}$ In cases of chronic hepatitis, the transmission routes appear to be the same as in the general population (low endemic areas), such as consumption of pork, game meat and shellfish. ${ }^{9,43}$

Due to the great variability in performance of serological tests and HEV RNA detection by RT-PCR mentioned above, it is difficult to determine the exact seroprevalence and incidence of chronic hepatitis $\mathrm{E}^{9}$ The prevalence of post-transplant hepatitis $E$ infection in a non-endemic area appears to be $1-2 \% .{ }^{6,44}$ When evaluating hepatitis E PCR-RNA among transplanted patients with increased liver enzymes, incidence is between 4.3 and $6.5 \% .{ }^{9}$ Based on available data, about $60 \%$ of transplant patients exposed to hepatitis $\mathrm{E}$ become chronic and within 2 years and $10 \%$ progress to cirrhosis. ${ }^{6}$ In Hannover (Germany) results from the Abbott serological test indicated that the seroprevalence of anti-hepatitis $E$ IgG antibodies was $4.4 \%$ in 226 liver transplanted patients. In addition, HEV RNA was detected in $2.9 \%$ of transplant recipient patients who had an increase in transaminases of unknown origin, whereas the test was not positive in any patient who had no elevation of transaminases. ${ }^{44,45}$

Reducing immunosuppression has reportedly led to spontaneous viral clearance in $1 / 3$ of patients. ${ }^{1,9}$ In addition, chronic hepatitis $\mathrm{E}$ was also susceptible to antiviral therapy. ${ }^{1}$ There are cases of chronic hepatitis E reported in transplanted patients that have required hepatic re-transplantation. Patients who do not reach viral clearance before re-transplantation may progress with recurrence of hepatitis $E$, associated with progressive chronic hepatitis. $^{9}$ In patients with chronic disease, transaminase levels are elevated, around $300 \mathrm{IU} / \mathrm{L}$, but much lower than the levels found in immunocompetent patients, which are around 1000-3000 IU/L. ${ }^{9}$ Moreover, it has been observed that the progression of fibrosis is faster in transplanted patients (liver or other organ) who have chronic hepatitis $\mathrm{E}$, leading to cirrhosis in 2-3 years. ${ }^{45,46}$ In addition, the progression to fibrosis is even faster than that observed in cases of hepatitis $C$ recurrence after liver transplantation. ${ }^{9}$

It should be noted that the detection of both anti-hepatitis E IgM and IgG antibodies may occur quite late in subjects being treated with triple immunosuppression (calcineurin, steroid and mycophenolate inhibitors), ${ }^{45}$ so that RNA-PCR should be used for diagnosis. A retrospective study of 85 transplant recipients who acquired hepatitis $\mathrm{E}$ showed that $32 \%$ were symptomatic at diagnosis. Among the symptoms were fatigue $(24 \%)$, diarrhea $(6 \%)$, arthralgia (5\%), abdominal pain ( $3 \%)$ and jaundice (1 patient). That study also reported that $66 \%$ of the cases evolved to chronic disease and in the other $34 \%$ the disease resolved without intervention. ${ }^{6}$ There is also a reported case in which the donor had hidden hepatitis $E$ and induced chronic hepatitis $E$ with rapidly progressive evolution to cirrhosis in the recipient, leading to death. ${ }^{6,9,43}$

Some authors have described chronicity predictive factors of hepatitis $E$ infection in transplanted patients. These include profoundly immunosuppressed patients with reduced levels of CD2, CD3, CD4, use of tacrolimus, lower serum levels of interleukin-1 receptor antagonist and interleukin-2 receptor, and increased serum concentration of chemokines involved in liver leukocyte recruitment, such as RANTES, MIP-1, MCP-1 and CXCL8. ${ }^{41,47,48}$ In a large multicenter study, only the use of tacrolimus and thrombocytopenia were independent predictive factors for chronic hepatitis $E$ infection in transplant recipients. ${ }^{42,47}$ Another observed point was that transplanted patients who developed chronic disease have had greater heterogeneity of "quasispecies" of HEV, in relation to those that have had spontaneous resolution. 48 Pischke et al. ${ }^{9}$ have reported that use of mycophenolate mofetil has been associated with clearance of HEV in cardiac transplant recipients, although these data need to be confirmed. Wang et al. ${ }^{6}$ have reported that corticoid does not interfere with virus replication, but that calcineurin inhibitors stimulate and 
mycophenolic acids inhibit viral replication. In addition, it has been shown that the combination of ribavirin with mycophenolic acid has a greater ability to inhibit replication of the HEV.

\section{Conclusions}

Hepatitis E is an important cause of acute viral hepatitis worldwide, being the main cause of hepatitis in some countries. Despite this, it still poses several challenges and is not fully understood, with many unanswered questions. It is probably underdiagnosed, mostly due to a lack of reliable diagnostic methods. In addition, it is a disease that is largely potentially preventable by simple hygiene and sanitary measures and caution in food intake. Moreover, it can be already treated with a medication that is available all over the world and which has been shown to improve the prognosis of affected patients who are usually immunosuppressed. Nevertheless, the need for further studies on pathogenesis and treatment is evident, as well as the development of more accurate diagnostic methods and new drugs. Collectively, the current and upcoming knowledge will facilitate diagnosis and proper management, thereby improving prognosis and avoiding complications.

\section{Conflict of interest}

The authors have no conflict of interests related to this publication.

\section{Author contributions}

Performed collection of the data and drafting of the article (JAAAG, KCK), conceived the study objectives, designed the study and performed critical revision of the article (DGBM, APJ, CAPI).

\section{References}

[1] Hoofnagle JH, Nelson KE, Purcell RH. Hepatitis E. N Engl J Med 2012;367: 1237-1244. doi: 10.1056/NEJMra1204512.

[2] Krawczynski K. Foreword. Hepatitis E virus. Semin Liver Dis 2013;33:1-2. doi: $10.1055 / \mathrm{s}-0033-1338119$.

[3] Chandra NS, Sharma A, Malhotra B, Rai RR. Dynamics of HEV viremia, fecal shedding and its relationship with transaminases and antibody response in patients with sporadic acute hepatitis E. Virol J 2010;7:213. doi: 10.1186/ 1743-422X-7-213.

[4] Meng XJ. From barnyard to food table: the omnipresence of hepatitis E virus and risk for zoonotic infection and food safety. Virus Res 2011;161:23-30. doi: $10.1016 /$ j.virusres.2011.01.016.

[5] Purcell RH, Emerson SU. Hepatitis E: an emerging awareness of an old disease. J Hepatol 2008;48:494-503. doi: 10.1016/j.jhep.2007.12.008.

[6] Murali AR, Kotwal V, Chawla S. Chronic hepatitis E: A brief review. World J Hepatol 2015;7:2194-2201. doi: 10.4254/wjh.v7.i19.2194.

[7] World Health Organization. The global prevalence of hepatitis E virus infection and susceptibility: a systematic review. Available from: http://apps. who.int/iris/bitstream/10665/70513/1/WHO_IVB_10.14_eng.pdf, accessed December 2010.

[8] Ahmad I, Holla RP, Jameel S. Molecular virology of hepatitis E virus. Virus Res 2011;161:47-58. doi: 10.1016/j.virusres.2011.02.011.

[9] Kamar N, Dalton HR, Abravanel F, Izopet J. Hepatitis E virus infection. Clin Microbiol Rev 2014;27:116-138. doi: 10.1128/CMR.00057-13.

[10] Unzueta A, Rakela J. Hepatitis E infection in liver transplant recipients. Liver Transpl 2014;20:15-24. doi: 10.1002/lt.23764.

[11] Sridhar S, Teng JLL, Chiu TH, Lau SKP, Woo PCY. Hepatitis E virus genotypes and evolution: emergence of camel hepatitis E variants. Int J Mol Sci 2017; 18:869. doi: $10.3390 /$ ijms 18040869 .

[12] Woo PC, Lau SK, Teng JL, Tsang AK, Joseph M, Wong EY, et al. New hepatitis E virus genotype in camels, the Middle East. Emerg Infect Dis 2014;20: 1044-1048. doi: 10.3201/eid2006.140140.
[13] Lee GH, Tan BH, Teo EC, Lim SG, Dan YY, Wee A, et al. Chronic infection with camelid hepatitis $E$ virus in a liver transplant recipient who regularly consumes camel meat and milk. Gastroenterology 2016;150:355-357.e3. doi: $10.1053 / j$.gastro. 2015.10.048.

[14] Wedemeyer H, Rybczynska J, Pischke S, Krawczynski K. Immunopathogenesis of hepatitis E virus infection. Semin Liver Dis 2013;33:71-78. doi: 10.1055/ $\mathrm{s}-0033-1338118$.

[15] Shrestha MP, Scott RM, Joshi DM, Mammen MP Jr, Thapa GB, Thapa N, et al. Safety and efficacy of a recombinant hepatitis E vaccine. N Engl J Med 2007; 356:895-903. doi: 10.1056/NEJMoa061847.

[16] Geng Y, Zhang H, Huang W, J Harrison T, Geng K, Li Z, et al. Persistent hepatitis e virus genotype 4 infection in a child with acute lymphoblastic leukemia. Hepat Mon 2014;14:e15618. doi: 10.5812/hepatmon.15618.

[17] Passos-Castilho AM, de Sena A, Reinaldo MR, Granato CF. Hepatitis E virus infection in Brazil: results of laboratory-based surveillance from 1998 to 2013. Rev Soc Bras Med Trop 2015;48:468-470. doi: 10.1590/00378682-0036-2015.

[18] Heldt FH, Staggmeier R, Gularte JS, Demoliner M, Henzel A, Spilki FR. Hepatitis E virus in surface water, sediments, and pork products marketed in Southern Brazil. Food Environ Virol 2016;8:200-205. doi: 10.1007/ s12560-016-9243-7.

[19] Kumar S, Subhadra S, Singh B, Panda BK. Hepatitis E virus: the current scenario. Int J Infect Dis 2013;17:e228-e233. doi: 10.1016/j.jij. 2012.11.026.

[20] Matsubayashi K, Kang JH, Sakata H, Takahashi K, Shindo M, Kato M, et al. A case of transfusion-transmitted hepatitis $\mathrm{E}$ caused by blood from a donor infected with hepatitis E virus via zoonotic food-borne route. Transfusion 2008:48:1368-1375. doi: 10.1111/j.1537-2995.2008.01722.x.

[21] Piper-Jenks N, Horowitz HW, Schwartz E. Risk of hepatitis E infection to travelers. J Travel Med 2000;7:194-199.

[22] Sridhar S, Lau SK, Woo PC. Hepatitis E: A disease of reemerging importance. J Formos Med Assoc 2015;114:681-690. doi: 10.1016/j.jfma.2015.02.003.

[23] Ahmed A, Ali IA, Ghazal H, Fazili J, Nusrat S. Mystery of hepatitis e virus: recent advances in its diagnosis and management. Int $\mathrm{J}$ Hepatol 2015;2015: 872431. doi: $10.1155 / 2015 / 872431$.

[24] Manka P, Bechmann LP, Coombes JD, Thodou V, Schlattjan M, Kahraman A, etal. Hepatitis Evirus infection as a possible cause of acute liver failure in Europe. Clin Gastroenterol Hepatol 2015;13:1836-1842.e2. doi: 10.1016/j.cgh.2015.04. 014.

[25] Davern TJ, Chalasani N, Fontana RJ, Hayashi PH, Protiva P, Kleiner DE, et al. Acute hepatitis $E$ infection accounts for some cases of suspected druginduced liver injury. Gastroenterology 2011;141:1665-1672.e9. doi: 10. 1053/j.gastro.2011.07.051

[26] Aggarwal R. Clinical presentation of hepatitis E. Virus Res 2011;161:15-22. doi: 10.1016/j.virusres.2011.03.017.

[27] Pavio N, Mansuy JM. Hepatitis E in high-income countries. Curr Opin Infect Dis 2010;23:521-527. doi: 10.1097/OCO.0b013e32833de683.

[28] Fujiwara S, Yokokawa Y, Morino K, Hayasaka K, Kawabata M, Shimizu T. Chronic hepatitis E: a review of the literature. J Viral Hepat 2014;21: 78-89. doi: $10.1111 /$ jvh. 12156

[29] Grewal P, Kamili S, Motamed D. Chronic hepatitis E in an immunocompetent patient: a case report. Hepatology 2014;59:347-348. doi: 10.1002/hep. 26636.

[30] Kamar N, Bendall RP, Peron JM, Cintas P, Prudhomme L, Mansuy JM, et al. Hepatitis E virus and neurologic disorders. Emerg Infect Dis 2011;17:173179. doi: $10.3201 /$ eid 1702.100856

[31] Kamar N, Weclawiak H, Guilbeau-Frugier C, Legrand-Abravanel F, Cointault O, Ribes $\mathrm{D}$, et al. Hepatitis $\mathrm{E}$ virus and the kidney in solid-organ transplant patients. Transplantation 2012:93:617-623. doi: 10.1097/TP.0b013e318245f14c.

[32] Drobeniuc J, Meng J, Reuter G, Greene-Montfort T, Khudyakova N, Dimitrova $Z$, et al. Serologic assays specific to immunoglobulin $M$ antibodies against hepatitis E virus: pangenotypic evaluation of performances. Clin Infect Dis 2010;51:e24-e27. doi: 10.1086/654801.

[33] Legrand-Abravanel F, Thevenet I, Mansuy JM, Saune K, Vischi F, Peron JM, et al. Good performance of immunoglobulin $\mathrm{M}$ assays in diagnosing genotype 3 hepatitis E virus infections. Clin Vaccine Immunol 2009;16:772-774. doi: 10.1128/CVI.00438-08.

[34] Kamar N, Rostaing L, Abravanel F, Garrouste C, Lhomme S, Esposito L, et al. Ribavirin therapy inhibits viral replication on patients with chronic hepatitis $e$ virus infection. Gastroenterology 2010:139:1612-1618. doi: 10.1053/j. gastro.2010.08.002

[35] Kamar N, Izopet J, Tripon S, Bismuth M, Hillaire S, Dumortier J, et al. Ribavirin for chronic hepatitis $\mathrm{E}$ virus infection in transplant recipients. N Engl J Med 2014;370:1111-1120. doi: 10.1056/NEJMoa1215246.

[36] Peters van Ton AM, Gevers TJ, Drenth JP. Antiviral therapy in chronic hepatitis E: a systematic review. J Viral Hepat 2015;22:965-973. doi: 10.1111/jvh. 12403.

[37] Dao Thi VL, Debing Y, Wu X, Rice CM, Neyts J, Moradpour D, et al. Sofosbuvir inhibits hepatitis $E$ virus replication in vitro and results in an additive effect when combined with ribavirin. Gastroenterology 2016;150:82-85.e4. doi: 10.1053/j.gastro.2015.09.011. 
[38] Zhang J, Shih JW, Wu T, Li SW, Xia NS. Development of the hepatitis E vaccine: from bench to field. Semin Liver Dis 2013;33:79-88. doi: $10.1055 / \mathrm{s}-0033-$ 1338116.

[39] Zhu FC, Zhang J, Zhang XF, Zhou C, Wang ZZ, Huang SJ, et al. Efficacy and safety of a recombinant hepatitis $E$ vaccine in healthy adults: a large-scale, randomised, double-blind placebo-controlled, phase 3 trial. Lancet 2010; 376:895-902. doi: 10.1016/S0140-6736(10)61030-6.

[40] Zhang J, Zhang XF, Huang SJ, Wu T, Hu YM, Wang ZZ, et al. Long-term efficacy of a hepatitis E vaccine. N Engl J Med 2015;372:914-922. doi: 10. 1056/NEJMoa1406011.

[41] Kamar N, Selves J, Mansuy JM, Ouezzani L, Péron JM, Guitard J, et al. Hepatitis $\mathrm{E}$ virus and chronic hepatitis in organ-transplant recipients. N Engl J Med 2008;358:811-817. doi: 10.1056/NEJMoa0706992.

[42] Im GY, Sehgal V, Ward SC. A case of undulating fevers and elevated liver tests after pancreas-kidney transplantation. Semin Liver Dis 2013;33: 89-93. doi: 10.1055/s-0033-1338117.

[43] Kamar N, Izopet J, Dalton HR. Chronic hepatitis e virus infection and treatment. J Clin Exp Hepatol 2013;3:134-140. doi: 10.1016/j.jceh.2013. 05.003
[44] Kamar N, Rostaing L, Izopet J. Hepatitis E virus infection in immunosuppressed patients: natural history and therapy. Semin Liver Dis 2013;33: 62-70. doi: 10.1055/s-0033-1338115.

[45] Pischke S, Suneetha PV, Baechlein C, Barg-Hock H, Heim A, Kamar N, et al. Hepatitis $E$ virus infection as a cause of graft hepatitis in liver transplant recipients. Liver Transpl 2010;16:74-82. doi: 10.1002/It.21958.

[46] Kamar N, Mansuy JM, Cointault O, Selves J, Abravanel F, Danjoux M, et al. Hepatitis E virus-related cirrhosis in kidney- and kidney-pancreas-transplant recipients. Am J Transplant 2008;8:1744-1748. doi: 10.1111/j.1600-6143. 2008.02286.x.

[47] Kamar N, Garrouste C, Haagsma EB, Garrigue V, Pischke S, Chauvet C, et al. Factors associated with chronic hepatitis in patients with hepatitis $\mathrm{E}$ virus infection who have received solid organ transplants. Gastroenterology 2011;140:1481-1489. doi: 10.1053/j.gastro.2011.02.050.

[48] Lhomme S, Abravanel F, Dubois M, Sandres-Saune K, Rostaing L, Kamar N, et al. Hepatitis $\mathrm{E}$ virus quasispecies and the outcome of acute hepatitis $\mathrm{E}$ in solid-organ transplant patients. J Virol 2012;86:10006-10014. doi: 10.1128/ JVI.01003-12. 